\title{
LITERATUR
}

\section{Europäisches Parlament und Parlamentarisierung der EU}

\author{
Daniela Kietz*
}

Das Mehrebenensystem der Europäischen Union durchlief seit der Gründung der Europäischen Gemeinschaft für Kohle und Stahl vor mehr als über 50 Jahren, vor allem aber in den letzten 20 Jahren einen stetigen, in seinem Ausmaß erstaunlich umfassenden Prozess der (Re-)Parlamentarisierung. Dieser lässt sich sowohl auf der nationalen als auch der europäischen Ebene nachweisen. In den über $50 \mathrm{Jah}-$ ren seines Bestehens hat sich das Europäische Parlament ${ }^{1}$ von einer mit wenigen Rechten ausgestatteten, vorrangig konsultativen Institution zu einem Hauptakteur der Europäischen Union (EU) mit weit reichenden Gesetzgebungs-, Kontroll-, und Budgetrechten im Machtdreieck mit dem Ministerrat und der Kommission entwickelt. Parallel zu diesem Parlamentarisierungsprozess des politischen Systems der EU stieg auch die wissenschaftliche Auseinandersetzung mit dem Europäischen Parlament. Heute liegt ein reichhaltiges Wissen zur seiner internen Funktionsweise und Entscheidungsfindung, der allgemeinen Entwicklung seiner Rechte, den Wahlen zum Europäischen Parlament und dem Verhalten von Abgeordneten und auch seiner Beziehung zu den beiden anderen Hauptakteuren, dem Ministerrat und der Kommission, vor. Nichtsdestotrotz bleiben wichtige Fragen offen und bedürfen weiterer Forschungsanstrengungen. ${ }^{2}$ Der vorliegende Beitrag setzt sich mit ausgesuchten Veröffentlichungen zum Europäischen Parlament auseinander, die einige dieser Lücken zu füllen versuchen, und liefert einen Ausblick auf den Stand der Forschung zur Rolle nationaler Parlamente, die einen
Berthold Rittberger (Hrsg.): Building Europe's Parliament. Democratic Representation beyond the Nation State, Oxford University Press: Oxford 2005, ISBN 0-19-927342-1; 240 Seiten, 74 \$.

Werner J. Patzelt (Hrsg.): Parlamente und ihre Macht. Kategorien und Fallbeispiele institutioneller Analyse, [Studien zum Parlamentarismus, Band 2], Nomos Verlagsgesellschaft: Baden-Baden 2005, ISBN 3-8329-1588-5; 305 Seiten, $34 €$.

Andreas Maurer und Dietmar Nickel (Hrsg.): Das Europäische Parlament. Supranationalität, Repräsentation und Legitimation, [Schriftenreihe des Arbeitskreises Europäische Integration e.V., Band 54], Nomos Verlagsgesellschaft: Baden-Baden 2005, ISBN 3-8329-1543-5; 291 Seiten, $55 €$.

Oskar Niedermayer und Hermann Schmitt (Hrsg.): Europawahl 2004, VS Verlag für Sozialwissenschaften: Wiesbaden 2005, ISBN 3-53114256-9; 170 Seiten, 22,90€.

Rudolf Hrbek (Hrsg.): European Parliament Elections 2004 in the Ten New EU Member States. Towards the Future European Party System, Nomos Verlagsgesellschaft: Baden-Baden 2005, ISBN 3-8329-1446-3; 276 Seiten, $69 €$.

zweiten wichtigen Legitimationsträger europäischer Entscheidungen darstellen.

\section{Der Machtaufstieg des Europäischen Par- laments}

Erstaunlicherweise bietet die Literatur zum Europäischen Parlament bis heute nur spärli-

\footnotetext{
Dipl.-Pol. Daniela Kietz, Forschungsgruppe EU-Integration, Stiftung Wissenschaft und Politik, Berlin.

Zunächst als Gemeinsame Versammlung der EGKS.

2 Siehe für einen umfassenden Stand der Forschung Simon Hix: Fifty Years On: Research on the European Parliament, Journal of Common Market Studies, 2/2003, S. 191-202.
} 
che Antworten auf eine der grundlegendsten Fragen: Wie erklärt sich die Einrichtung und Kompetenzerweiterung des Europäischen Parlaments? Weshalb schufen die sechs Gründerstaaten die Gemeinsame Versammlung, den Vorläufer des heutigen Europäischen Parlaments, und versahen es im Verlaufe der letzten 50 Jahre mit Legislativ-, Kontroll-, und Budgetrechten, die denen der nationalen Parlamente kaum nachstehen? Als Herren der Verträge sind die Mitgliedstaaten ermächtigt, europäisches Primärrecht zu verändern, und somit waren sie allein es, die dem Parlament sukzessiv immer mehr Rechte übertragen haben. Ein großer Teil der Literatur zu den Internationalen Beziehungen im Allgemeinen und zur Europäischen Integration im Besonderen legt mittels funktionaler Argumente dar, dass unter bestimmten Bedingungen rational und nutzenorientiert agierende Staaten internationale Organisationen schaffen und selektiv Kompetenzen an diese delegieren, um effiziente Lösungen für Probleme des kollektiven Handelns in der zwischenstaatlichen Kooperation voranzutreiben. Während diese Argumente im Rahmen der Europäischen Gemeinschaft auf die Delegation von Kompetenzen an den EuGH, die Kommission oder unabhängige Agenturen anwendbar sind, erklären sie nicht, warum die Mitgliedstaaten ein weithin unabhängiges, supranationales Parlament mit weitreichenden Kompetenzen ausstatten sollten. Denn sie können dessen Besetzung und Verhalten nicht kontrollieren, und die Partizipation des Europäischen Parlaments am Gesetzgebungsprozess bringt keine unmittelbaren Effizienzgewinne, sondern, ganz im Gegenteil, einen Machtverlust der Mitgliedstaaten mit sich.

Unter Rückgriff auf Annahmen des soziologischen Institutionalismus bestätigt Berthold Rittberger in „Building Europe's Parliament“ jenen Strang der Forschung, der argumentiert, dass politische Eliten die Entscheidung bei der Einrichtung und kompetenzrechtlichen Ausstattung von Institutionen im Rahmen zwischenstaatlicher Kooperation nicht nur durch einen erhofften materiellen Mehrwert
(Effizienzsteigerung der wirtschaftlich oder sicherheitspolitisch motivierten Kooperation), sondern auch durch normative Standards , angemessenen' Regierens geleitet werden.

Im Kern argumentiert der Autor wie folgt: Die auf europäischer Ebene agierenden politischen Eliten sind geprägt von nationalen Verständnissen und Standards angemessenen Regierens. Einer der zentralen und unumstrittenen Werte ist die demokratische Legitimation politischer Entscheidungen. An diesen Standards, so das Argument, muss sich auch die Entscheidungsfindung auf europäischer Ebene messen, dass heißt, die Standards werden aus dem nationalen Rahmen auf die europäische Ebene übertragen. Nach Rittberger trägt jeder Souveränitätstransfer - die Abgabe von Legislativkompetenzen an die europäische Ebene und der Übergang von der Einstimmigkeit zu Mehrheitsentscheidungen im Ministerrat - in der Regel zwar zu einer Steigerung der Outputlegitimität, dass heißt zur Effizienz der getroffenen Entscheidungen bei. Er entbehrt jedoch durch das Umgehen nationaler parlamentarischer Beschlussfassungswege und im Falle der Nichtbeteiligung des Europäischen Parlaments der nötigen Inputlegitimität.

Laut Rittberger sind sich politische Eliten über das aus dieser Asymmetrie hervorgehende Legitimationsdefizit durchaus bewusst. Um die Defizite zu beheben initiieren sie Reformen, nämlich die Ausweitung der Kompetenzen des Europäischen Parlaments und seit dem Maastrichter Vertrag verstärkt auch der nationalen Parlamente. Die Ansichten darüber, ob eher die Stärkung der nationalen Parlamente oder die des Europäischen Parlaments die Inputlegitimation europäischer Entscheidungen erhöht, hängen dabei mit den unterschiedlichen, national geprägten Vorstellungen darüber zusammen, welche Institutionen und Prozesse Regieren auf supranationaler Ebene legitimieren sollten. Der Autor greift hier konkret drei in der Wissenschaft etablierte idealtypische Legitimationsvorstellungen auf: intergouvernementale Kooperation, 
Föderalstaat und Wirtschaftsgemeinschaft. Hauptträger dieser Legitimationsvorstellungen sind die politischen Parteien, dass heißt nicht der Mitgliedstaat als Ganzes, sondern die Regierungsparteien in ihrem nationalen Interaktionsfeld sind Objekt der Analyse. Verfechter der Idee des Föderalstaates, die vor allem von den deutschen Regierungsparteien seit Gründung der europäischen Institutionen vertreten wurde, setzen sich entsprechend für eine starke, direkt legitimierte parlamentarische Vertretung auf europäischer Ebene ein. Anhänger des intergouvernementalen Konzeptes hingegen sehen Legitimität weiterhin im Nationalstaat verankert und auf europäischer Ebene durch den indirekt legitimierten Ministerrat gewährleistet.

In drei historischen Fallstudien, in denen dem Parlament jeweils ein grundlegendes parlamentarisches Recht übertragen wurde - die Schaffung der Gemeinsamen Versammlung durch den Vertrag über die Gründung der Gemeinschaft für Kohle und Stahl 1951 mit dem Kontrollrecht gegenüber der Hohen Behörde als dem Vorläufer der Kommission; die Übertragung von Budgetrechten durch den Vertrag von Luxemburg 1970 und das Recht zur Mitwirkung an der Gesetzgebung durch die Einführung des Kooperationsverfahrens durch die Einheitliche Europäische Akte 1986 zeigt der Autor, dass Auslöser der Übertragung von Rechten an das Europäische Parlament jeweils die Souveränitätsübertragung an die europäische Ebene und die damit einhergehenden Legitimitätsbedenken der mitgliedstaatlichen Akteure waren.

Das Hauptargument sowie die Verknüpfung mit den national geprägten Legitimationsvorstellungen ist einleuchtend und überzeugend dargestellt, wenn auch im Kern nicht neu. ${ }^{3}$ Die theoretische Herleitung, die Klarheit des Arguments und vor allem seine dokumentenbasierte Überprüfung anhand der drei ausgesuchten Fälle ist plausibel. Dennoch bleiben wichtige Fragen offen. So ändert sich zwischen dem ersten untersuchten Fall von 1951 und dem zweiten Fall 1970 die Position der Regierungen der Beneluxstaaten bezüglich einer Stärkung des Europäischen Parlaments fundamental. Was bewirkte diese Änderung? Was passierte in dem Zeitfenster zwischen diesen beiden Fällen? Da es sich bei Leitbildern um über lange Zeit gewachsene Wertzuweisungen handelt, kann eigentlich nicht von einer kurzfristigen Veränderung ausgegangen werden. Entsprechend müsste ein Wechsel der Regierungsparteien vermutet werden oder die Koexistenz von mehreren Legitimationsvorstellungen. Diese Frage bleibt gänzlich unbeantwortet, wie auch diejenige nach der ,Stickiness' der Legitimitätsvorstellungen, auf die im theoretischen Teil nicht sehr vertieft eingegangen wird. Des Weiteren stellt sich die Frage, ob die drei nebeneinander gestellten Fälle wirklich unabhängig voneinander sind. Während das Hauptargument sehr gut bei der Erklärung der Einrichtung einer parlamentarischen Versammlung greift, stellt sich bereits 1986 die Frage, ob nicht gleichzeitig das Parlament als Akteur selbst eine aktive Strategie der Ausweitung seiner Kompetenzen verfolgte, die nicht nur auf dem Versuch gründete, die Mitgliedstaaten argumentativ von der notwendigen Stärkung seiner Kompetenzen zur Legitimation der getroffenen Entscheidungen zu überzeugen, sondern auch auf dem Rückgriff auf andere Machtressourcen? Anders: Hat sich das Parlament die ihm 1970 und 1975 übertragenen Budgetrechte als Machtressource zu Nutze gemacht, um sich beispielsweise über die Haushaltsverweigerung weitere Rechte zu erstreiten? Eine solche aktive Rolle des Europäischen Parlaments in der Ausweitung seiner Kompetenzen steht der Argumentation Rittbergers nicht entgegen, sondern ergänzt sie. Der Machtaufstieg des Parlaments kann sehr gut auf Grund von Legitimationsbedenken der politischen Eliten und die dadurch ausgelöste Übertragung neuer Rechte an das Europäische Parlament im Ver-

3 Siehe dazu unter anderem Andreas Maurer: Parlamentarische Demokratie in der Europäischen Union. Der Beitrag des Europäischen Parlaments und der nationalen Parlamente, Baden-Baden 2002. 
laufe von Vertragsreformen als auch über Machtkämpfe abgerungene Kompetenzen stattgefunden haben.

Einen vergleichenden Einblick in den Machtaufstieg und -erhalt von Parlamenten bieten die Beiträge in dem von Werner $G$. Patzelt herausgegebenen Sammelband „Parlamente und ihre Macht" auf der Grundlage eines einheitlichen Konzeptes zu Entstehung und Entfaltung institutioneller Macht. Allein schon die vergleichende Perspektive bringt einen großen Mehrwert. Denn allzu oft wird das Europäische Parlament als weder mit nationalen Parlamenten noch mit den parlamentarischen Versammlungen internationaler Organisationen vergleichbares Unikum behandelt. Wertvolle Einsichten aus einer systematisch vergleichenden Analyse bleiben dadurch verwehrt. Stephan Dreischer führt in seinem Beitrag vor Augen, wie das Europäische Parlament über so genannte institutionelle Machtmechanismen im gemeinsamen Handlungsraum von Kommission, Parlament und Rat seine eigenen Kompetenzen in den letzten 20 Jahren zu Lasten der beiden anderen Institutionen ausdehnte. Interessant ist die historisch fundierte Feststellung, dass der Machtaufstieg des Europäischen Parlaments keine Ausnahme, sondern ,eine tendenziell typische Variante der Parlamentsgenese in freiheitlich-demokratischen Systemen ist". Nach Ansicht des Autors behandeln Analysen der Regierungskonferenzen - die die formalen Rechte des Europäischen Parlaments ändern - in der Regel nur einen Teil der Prozesse, die bei einem parlamentarischen Machtaufstieg zu Gange sind, „,[d]enn selten wird ein Parlament allein dadurch machtvoll, dass es sich auf die freiwillige Machtreduktion von mit ihm in Konkurrenz stehenden Institutionen [mit anderen Worten der Ministerrat, Anmerkung der Autorin] verlässt. Meist wird es vielmehr so sein, dass ein Parlament seine Machtposition in mehr oder minder schweren, verdeckt oder offen ausgetragenen Kämpfen wird erringen müssen“. Hier liegt also eine andere Akteursperspektive als im oben diskutierten Band vor: Wie beeinflusst das Europäische Parlament selbst den Ausbau seiner Kompetenzen? Wie verstetigen sich solche oftmals informellen Praktiken und wann werden sie in den ,,von allen Akteuren akzeptierten und getragenen Werte- und Normenkanon als ,gemeinsamer' Handlungsraum verankert werden?" Anders ausgedrückt: Wann werden vom Parlament auf diese Weise errungene Kompetenzen im Dreieck RatKommission-Parlament allgemein akzeptiert und nicht mehr in Frage gestellt? Der Autor stellt nicht in Frage, dass Legitimitätsbedenken der politischen Eliten der Hauptgrund sind, weshalb sie dem Parlament überhaupt nach und nach weit reichende Rechte übertragen haben. Nichtsdestotrotz ist das Parlament selbst ein Akteur mit dem strategischen Interesse der eigenen Machtausweitung. Wie geht es dabei vor?

Als Machtmechanismus gelten Dreischer formelle und informelle institutionelle Regeln die dem Parlament als Hebelwerke dienen, um seine Macht im strategischen Handlungsraum mit Rat und Kommission zu vergrößern. Diesen Mechanismen ist gemeinsam, dass sie interinstitutionell wirken, das Eintreten einer erwarteten Verhaltensweise der anderen Institution garantieren und jederzeit und transpersonell wirken. Grundvoraussetzung für die Herausbildung solcher Machtmechanismen ist ein Mindestmaß an Einfluss auf die getroffenen Entscheidungen als Verhandlungspotenzial. Als Beispiele für die Entstehung solcher Machtmechanismen zieht der Autor den Fall des Mistrauensvotums und der Einzelanhörungen der Kommissare bei der Einsetzung einer neuen Kommission heran. Während ersteres durch die Staats- und Regierungschefs ins Primärrecht eingeführt wurde, handelt es sich bei den Einzelanhörungen um eine auf informeller Basis im Geschäftsordnungsrecht des Parlaments etablierte Regelung, die bis heute nicht im Vertragswerk vorgesehen ist, aber von allen designierten Kommissionsmitgliedern vor der Drohkulisse einer möglichen Ablehnung der Kommission befolgt wird. Insbesondere das Mistrauensvotum ist ein Beispiel für eine Machtkompetenz des Parla- 
ments, die auf Grund seiner inneren Organisation und Spaltung oft ungenutzt blieb und erst seit der erfolgreichen Machtprobe mit der Kommission unter Präsident Santer ein ernst genommenes Sanktionsmittel darstellt. Oftmals werden Machtmechanismen erst durch solche Machtkämpfe zu wirksamen Machtinstrumenten gegenüber der Kommission und dem Rat - im Fall der Einzelanhörungen war dies die erfolgreiche Auseinandersetzung mit dem designierten Präsidenten Barroso über die Zusammensetzung der Kommission - in denen das Parlament den Willen zeigt, die ihm zur Verfügung stehenden Kontroll- und Sanktionsmöglichkeiten auch zu nutzen. Dennoch zeigt der Beitrag von Dreischer, dass solche Mechanismen meist nur wirksam sein können, wenn sie durch das gesamte Parlament gestützt werden. Diese Einheit ist jedoch aufgrund seiner internen Organisationen, insbesondere der nicht nur zwischen den Fraktionen sondern auch Nationen verlaufenden Spannungslinien, nur schwer herzustellen. Daher waren bisher viele erfolgreiche Machtproben, aus denen die nötige Verlässlichkeit eines Machtmechanismuses resultierte, einer Verkettung günstiger Bedingungen zu verdanken, wie einem schwachen Kommissionspräsidenten und politisch höchst umstrittenen Kommissionskandidaten.

Aus einer ähnlichen Perspektive arbeiten einige Beiträge in dem von Andreas Maurer und Dietmar Nickel herausgegebenen Sammelband „Das Europäische Parlament. Supranationalität, Repräsentation und Legitimation" die Rolle des Europäischen Parlaments in der institutionellen und materiell-rechtlichen Ausgestaltung der Union im Rahmen von formalen Vertragsreformprozessen heraus. Die Beiträge von Große Hüttmann und Schunz attestieren dem Parlament in den Vertragsreformprozesses seit Maastricht parallel zu seinem großen Bedeutungszuwachs in der Gesetzgebung eine zunehmend ,reale Befugnis [...] zur Teilnahme an Vertragsreformprozessen." Sie zeigen, wie das Europäische Parlament auch ohne formales Recht zur Teilnahme an der Verhandlungen oder zur
Ratifizierung der Verträge bei Vertragsreformen die Rolle eines Systemgestalters einnimmt. Große Hüttmann zeigt im historischen Überblick wie der Einfluss des Europäischen Parlaments auf die Systemgestaltung mit jeder Regierungskonferenz konkreter wurde und viele der Neuerungen der Verträge wie die Unionsbürgerschaft oder die vom Verfassungsvertrag vorgesehene Systematisierung der Verträge ihren Ursprung in parlamentarischen Ausschüssen und Expertenrunden hatten. Der Beitrag von Schunz unterstreicht, dass sich die Organisation der Entscheidungsfindung im Konvent zumindest in einigen Phasen noch günstiger auf die Einflussmöglichkeiten des Parlaments auswirkte. Dies ist vor allem dort der Fall, wo die Reformberatungen parlamentarischen Regeln folgen und Raum für Überzeugung durch Argumentation ist, wie streckenweise im Falle des Konvents und Reflexionsgruppen vorheriger Regierungskonferenzen. Der Einfluss des Europäischen Parlaments ist besonders in den Bereichen hoch, in denen es sich eindeutig Expertise erarbeitet hat und ein Eigeninteresse verfolgt. Das gilt für die Ausgestaltung der Legislativverfahren und wenn es ihm gelingt, Koalitionen von Mitgliedstaaten hinter seiner Position zu vereinigen und gemeinsam erfolgreich zu sein. Wenig Einfluss hat das Europäische Parlament nach wie vor bei Themen, in denen Mitgliedstaaten sich gegen eine substanzielle Einflussnahme supranationaler Institutionen wehren, wie in außenpolitischen Fragen und in stark intergouvernemental geprägten, informellen Verhandlungsphasen wie der Endphase der Konventsberatungen.

In Ergänzung zur Untersuchung der Rolle des Parlaments bei formalen Vertragsreformen zeigt der Beitrag von Maurer und Kietz auf welche Weise das Europäische Parlament unterhalb dieser Ebene durch den Rückgriff auf informelle Mechanismen seinen Kompetenzen auszuweiten vermag. Anhand der informellen Interinstitutionellen Vereinbarungen (IIV) zwischen Rat und Parlament über die Finanzierung der Gemeinsamen Außen- und Sicherheitspolitik aus dem Jahr 1997 zeigen 
sie, wie das Europäische Parlament selbst in intergouvernementalen Bereichen seine vertraglichen Informations- und Kontrollrechte präzisieren und ausweiten kann. Obwohl streng genommen nur politisch verbindlich, etablieren und institutionalisieren IIV in der informellen Arena Rechte des Europäischen Parlaments und schaffen Pfadabhängigkeiten, die in einigen Fällen sogar später in Vertragsreformen münden. Die Autoren zeigen, dass das Parlament über eine Reihe von Möglichkeiten verfügt - und diese auch strategisch einsetzt - den Rat und die Kommission zu überzeugen, die von ihm initiierten IIV abzuschließen. Dazu gehört vor allem der Rückgriff auf seine Budgetrechte, die Möglichkeit der Ablehnung des Haushaltes oder der Verweigerung von Finanzmitteln, eine Gesetzesvorlage zu verzögern und im Zweifelsfall sogar Gesetzesvorhaben im Rahmen des Mitentscheidungsverfahrens abzulehnen. Hinzu kommen die Kontrollrechte gegenüber der Kommission. Über diese ,Bargaining Chips' gelingt es dem Parlament, den Rat und die Kommission in interinstitutionelle Konflikte und Machtkämpfe zu verwickeln. Die Tatsache, dass die Europaparlamentarier über einen längeren Zeithorizont verfügen als nationale Regierungen und durch ihre geringe Wahlkreisbindung nicht im dem selben Maße wie die Regierungen darauf angewiesen sind, kurzfristige Ergebnisse zu erzielen, spielt für den Erfolg des Parlaments keine unbedeutende Rolle.

Weitere Beiträge des Sammelbandes spiegeln unter anderem den aktuellen Stand der Forschung zur Rolle des Parlaments für die Interessenvertretung und das Lobbying auf europäischer Ebene sowie dessen Einfluss in zentralen Politikbereichen der EU wider. Anlässlich der Europawahl sollte in dem Band eine Bestandsaufnahme der Kompetenzentwicklung des Europäischen Parlaments sowie Analysen zur Wahrnehmung der parlamentarischen Funktionen, zum Europa-Wahlkampf sowie den Herausforderungen für das Parlament in der neuen Legislaturperiode vorgelegt werden. Mit besonderem Gewinn zu lesen sind sowohl die theoriegeleiteten Texte als auch die Beiträge aus der Praxis, die Einblicke in die Funktionsweise des Parlaments bieten. Das Buch wendet sich sowohl an das akademische Publikum als auch an die Praktiker in Regierung und Verwaltung. Angesichts der breit angelegten Thematik bleibt jedoch fast notgedrungen die Kohärenz des Bandes etwas auf der Strecke.

\section{Die (Neben-)Wahlen zum Europäischen Parlament}

Der von Oskar Niedermayer und Hermann Schmitt herausgegebene Sammelband „Europawahl 2004“" widmet sich der detaillierten Analyse des Ablaufes und der Ergebnisse der Wahl in Deutschland und stellt vor allem eine Frage: Handelte es sich bei den letzten Wahlen wieder einmal um nationale Test- oder Nebenwahlen? Die Prominenz des Verfassungskonvents und die Brisanz der kurz zuvor erfolgten Osterweiterung gaben Hoffnung, dass bei diesen Wahlen endlich europäische Themen hoch im Kurs stehen könnten. Niedermayer kommt in seiner Analyse des Wahlkampfes in Deutschland zu einem klaren Ergebnis. Wie auch bei vorangegangenen Europawahlen standen vor allem nationale Themen und Persönlichkeiten im Vordergrund des zum großen Teil innenpolitisch ausgerichteten Wahlkampfes. Weder war der Wahlkampf auf publikumswirksame europäische Spitzenkandidaten zugeschnitten, noch wurden europäische Themen besonders kommuniziert. Einen wirklich ,europäischen Wahlkampf hatten nur die FDP und Bündnis 90/Die Grünen betrieben. Die FDP habe ihre Spitzenkandidatin von Anfang an in den Vordergrund ihres Wahlkampfes gerückt. Zudem wurde der Entwurf des Vertrags über eine Verfassung für Europa als ein zentraler und aktueller Aspekt europäischer Politik zum Wahlkampfthema erhoben. Die Grünen waren als einzige Partei mit einem europaweit einheitlichen Wahlprogramm angetreten und platzierten traditionell europäische Themen wie Umwelt- und Verbraucherschutz ganz oben auf der Wahlkampfliste. Kritisch zu be- 
trachten seien hingegen die tendenziell populistische Züge aufweisenden Wahlkämpfe von CSU und PDS. Niedermayer unterstreicht gleichzeitig, dass nicht nur die Parteien, sondern vor allem auch die deutschen Medien ihre Rolle im Wahlkampf kritisch zu prüfen hätten. Während diese den Parteien häufig die Fokussierung auf die nationale politische Arena vorwarfen, hätten sie selbst politische Entwicklungen auf der europäischen Ebene und auch die Kandidaten für das Europäische Parlament nur in ungenügendem Maße vorgestellt. Der Band enthält weitere, allesamt hoch informative und durch die Auswertung umfangreichen Datenmaterials fundierte Beiträge, nicht zuletzt die ausführliche Analyse der deutschen Wahlbeteiligung durch Hermann Schmitt. Dieser sieht ein weiteres Mal den Charakter der Europawahl als Nebenwahl bestätigt mit den typischen Merkmalen: Erstens eine niedrige Wahlbeteiligung auf Grund der als gering wahrgenommenen Bedeutung der Wahlen, der Enthaltung in Folge der Unzufriedenheit mit der nationalen Politik, aber auch einer kritischen Haltung gegenüber der EU vor dem Hintergrund der Osterweiterung und der damit verbundenen ökonomischen Ängste. Zweitens die Abstrafung der aktuellen Regierungsparteien, allerdings gewannen die Grünen aus oben angedeuteten Gründen Sitze hinzu.

$\mathrm{Zu}$ ähnlichen Ergebnissen kommen die Analysen der Wahlen in den zehn neuen Mitgliedstaaten in dem von Rudolf Hrbek herausgegebenen Buch „The European Parliament Elections 2004 in the Ten New Member States". Dessen großer Nutzen liegt vor allem in der einem einheitlichen Raster folgenden Analyse und in der umfassenden Datensammlung zu den ersten Europawahlen in diesen Ländern. Besonders die Darstellung der Einstellungen der wichtigsten Parteien zur Europäischen Union in einem Band und die Analyse der Einordnung der Parteien der einzelnen Länder in die europäischen Parteifamilien ist eine Fundgrube für weitere Forschungen. Hrbek kommt zusammenfassend zu dem Ergebnis, dass auch in den neuen Mit- gliedstaaten die Wahl des Europäischen Parlaments alle Merkmale einer Nebenwahl aufwies. Die Wahlbeteiligung war in den meisten Ländern extrem gering, die Regierungsparteien erlitten in fast allen Fällen hohe Verluste, während Kleinparteien und sogar völlig neue politische Gruppierungen hohe Ergebnisse einstrichen. Die Parteien investierten relativ wenig Ressourcen in den Wahlkampf, der von nationalen politischen Eliten und Inhalten dominiert wurde. Bedauerlicherweise fehlen, abgesehen von der kurzen Einleitung durch den Herausgeber, analytisch vergleichende Querschnittsanalysen. Man hätte sich diese beispielsweise sehr gut zu Themen wie Populismus oder Euroskepsis der Parteien oder dem Wählerverhalten vorstellen können.

Beide Sammelbände bestätigen die Kontinuität hinsichtlich der Natur der Wahl zum Europäischen Parlament, der Wahlkampfmechanismen und des Wählerverhaltens. Die, alte' und die ,neue' EU waren insofern schon kurz nach dem Beitritt der zehn neuen Mitgliedstaaten geeinter als man vermutet hätte. Weder die Analysen der Wahl in den alten noch in den neuen Mitgliedstaaten spiegeln das wider, was in den oben besprochenen Abhandlungen attestiert wurde: Dass sich nämlich das Europäische Parlament in den letzten zwanzig Jahren zu einem einflussreichen Akteur in der europäischen Arena entwickelt hat und damit zumindest theoretisch auch die Wahlen wesentlich an Bedeutung gewonnen haben.

\section{Neue Impulse für die Forschung zu den na- tionalen Parlamenten}

Ein zweiter größerer Strang der Forschung zur Rolle parlamentarischer Akteure in der Union befasst sich mit den nationalen Parlamenten. Während sich bis Ende der 1990er Jahre nur wenige Veröffentlichungen zu diesem Thema fanden, setzte spätestens mit der Erklärung von Laeken in Vorbereitung auf den Europäischen Konvent und der neuerlichen Diskussion über Maßnahmen zur Behebung des wahrgenommenen Demokratiedefizits in der EU eine Flut von Veröffentlichungen zu diesem Thema ein, jedoch ohne 
damit wesentlich neue Erträge für die Forschung zu liefern. Die vorliegende Literatur ist vor allem dadurch gekennzeichnet, dass zumeist nur einige wenige mitgliedstaatliche Parlamente (zu den üblichen Verdächtigen gehören Deutschland, Dänemark, Frankreich und Großbritannien) und oftmals nur die formalen Rechte der Parlamente und nicht deren wirkliche Anwendung untersucht werden. ${ }^{4}$ Die Diskrepanz zwischen dem formal Möglichen und dem real Praktizierten ist in vielen Parlamenten jedoch so groß, dass die Erklärung dieses Phänomens einen wichtigen Teil der Forschung in diesem Bereich ausmachen sollte. Das Gegenteil ist aber der Fall. Zudem steht weiterhin die allgemeine Aktivität der Parlamente im Bereich der EU-Politik im Vordergrund, politikfeldspezifische Analysen existieren kaum. Auch zu den Determinanten des Ausmaßes nationalparlamentarischer Beteiligung in EU-Angelegenheiten gibt es nur wenige empirisch gesättigte Forschungsergebnisse. ${ }^{5}$ Eine Forschungsagenda für Untersuchungen der Rolle und Aktivitäten nationaler Parlamente in der EU, die wirklich noch einen neuen Beitrag zur Debatte leisten möchten, sollte daher vor allem folgende Aspekte berücksichtigen: Welche Faktoren beeinflussen das Ausmaß und die Ausgestaltung der Einflussmöglichkeiten eines Parlamentes in EU-Angelegenheiten (der Euroskeptizismus der Parteien, das Regierungssystem, die Rollenbilder der Abgeordneten)? Unterscheidet sich die parlamentarische Aktivität in verschiedenen Politikbereichen; sind beispielsweise Parlamente im Bereich der politisch sensiblen und gleichzeitig hoch dynamischen europäischen Innen- und Justizpolitik aktiver als in weniger brisanten Bereichen? Variiert die Aktivität nach Entscheidungsmodus auf europäischer Ebene? Sind Parlamente dort aktiver wo das Europäische Parlament keine Einflussmöglichkeiten besitzt und sie selber noch Hauptlegitimationsträger politischer Entscheidungen sind, wie im Bereich der dritten Säule oder im Bereich der neuen Offenen Methode der Koordinierung? Welche Prozeduren nationalparlamentarischer Beteiligung haben die neuen Mitgliedstaaten eingerichtet und aus welchen Gründen? Welche Rolle spielt die interparlamentarische Kooperation im Rahmen der Europäisierung nationaler Parlamente? Antworten auf diese Fragen sollten einige überraschende Forschungsergebnisse zu Tage fördern.

\section{Ausblick}

Eine noch wesentlich umfangreichere Forschungsagenda ließe sich für das Europäische Parlament erstellen. Neben den andauernden Themen wie der Kompetenzentwicklung des Europäischen Parlaments im Machtgefüge, das es mit Rat und Kommission bildet, stehen vor allem Aspekte der internen Organisation und Funktionsweise im Vordergrund neuerer Forschung: wie wirkt sich die Osterweiterung, das heißt die Integration neuer Parteien in die Gruppierungen im Europäischen Parlament auf die interne Organisation und die Koalitionsbildung im Parlament aus? Ist eine steigende Politisierung der Entscheidungen festzustellen, und wie geht das Parlament mit seiner weiterhin wachsenden Teilnahme am Gesetzgebungsprozess in mehr und mehr Politikbereichen und den damit verbundene Herausforderungen an seine Leistungskapazitäten um? Fest steht, dass die Evolution des Parlaments im sich andauernd verändernden Institutionengefüge der Union ein noch lange nicht abgeschlossener Prozess ist. Es dürfte sich daher weiterhin zumindest eines regen akademischen Interesses erfreuen.

$4 \mathrm{Zu}$ den Ausnahmen und Standardwerken, die alle alten Mitgliedstaaten abdecken, gehört weiterhin, Andreas Maurer/ Wolfgang Wessels: National Parliaments on their Way to Europe. Losers or Latecomers?, Baden-Baden 2001.

5 Eine bemerkenswerte Ausnahme: Tapio Raunio: Holding governments accountable in European affairs: Explaining cross-national variation, in: Journal of Legislative Studies Nr. 3-4/2005, S. 319-342. 